EDITORIAL

\title{
To Close or not to Close PFOs in Cryptogenic Stroke, an Evolving Question
}

\author{
Alex Felix ${ }^{1,2,4}$ and Monica Luiza de Alcantara ${ }^{2,3} \odot$ \\ Instituto Nacional de Cardiologia, ${ }^{1}$ Rio de Janeiro, RJ - Brazil \\ Americas Medical City, ${ }^{2}$ Rio de Janeiro, RJ - Brazil \\ Rede D'Or São Luiz, ${ }^{3}$ Rio de Janeiro, RJ - Brazil \\ Diagnósticos da América, Rio de Janeiro, RJ - Brazil \\ Editorial related to the article: The Role of Patent Foramen Ovale Closure in the Secondary Prevention of Cryptogenic
}

A matter of intense debate in the last decades, the ideal strategy for secondary prevention in patients with cryptogenic stroke and patent foramen ovale (PFO) is not yet clearly defined. PFOs are prevalent in the general population and can be found in up to $40 \%$ of patients with cryptogenic stroke. ${ }^{1}$ Although in some cases of cryptogenic stroke PFO might be just an innocent bystander, paradoxical embolism is a wellknown potential causative mechanism, especially in patients with concomitant deep venous thrombosis, whereby PFO closure may represent an effective measure to avoid recurrences. ${ }^{2}$ Even among patients with $\mathrm{PFO}$, phenotypical heterogeneity may also determine percutaneous treatment suitability and directly impact on results, whereas the presence of large shunts, atrial septal aneurysm or associated complex septal defects must also be taken into account.

In the past years, several evidence-based guidelines and expert consensus on the management of patients with PFO and prevention of recurrent stroke have been published by international scientific societies, including a position paper sponsored by the European Association of Percutaneous Cardiovascular Interventions (EAPCI), with the participation of eight European scientific societies. ${ }^{3}$ A recent guideline by the American Academy of Neurology (AAN) was published online last April. ${ }^{4}$

This issue of the International Journal of Cardiovascular Sciences presents the results of an interesting study entitled "The Role of Patent Foramen Ovale Closure in the Secondary Prevention of

\section{Keywords}

Patent Foramen Ovale; Secondary Prevention; Stroke/ prevention and control; Risk Factors; Atrial Fibrillation.
Cryptogenic Stroke: a Meta-Analysis Report", authored by Pereira and coworkers. ${ }^{5}$ This study sheds new light on the discussion of this important topic, adding relevant information about the results of different management strategies for secondary prevention of stroke in patients with PFO. The authors included data from six randomized clinical trials with a total of 3,750 patients. Unlike other meta-analyses published so far, they considered only original clinical trial data, a difference that provides more reliability to the obtained results. Interestingly, there was a significant lower incidence of stroke in patients submitted to PFO closure compared with those who received medical treatment alone (risk ratio $[R R]=0.37 ; 95 \%$ confidence interval $[0.17$ to 0.78$]$; $\mathrm{p}=0.01$ ), in accordance with recent findings of the CLOSE and DEFENSE-PFO trials, ${ }^{6,7}$ which recruited only PFO patients with high-risk anatomic features.

It is important to emphasize that ischemic stroke may be caused by a variety of heterogeneous mechanisms, and adequate secondary prevention must focus on the correct target population. Not every PFO is deemed for closure and not every PFO anatomy is amenable to percutaneous closure, but once the procedure is correctly indicated, it is of paramount importance to assemble a multidisciplinary team in charge of choosing the correct device and minimizing complications. ${ }^{8}$ Diagnosing PFO is not always a simple matter. Many factors can contribute for a non-diagnostic study and yield false positive results, such as intrapulmonary shunts or false negative diagnoses due to an inadequate Valsalva maneuver or a redundant Eustachian valve, which may prevent the contrast solution from reaching the atrial septum. Therefore, in order to achieve the best results, it is crucial to follow a stepwise approach with transthoracic echo (TTE), transcranial Doppler (TCD)

Mailing Address: Alex Felix

Rua das Laranjeiras, 374. Postal Code: 22240-006, Rio de Janeiro, RJ - Brazil

E-mail: alexsfelix@gmail.com 


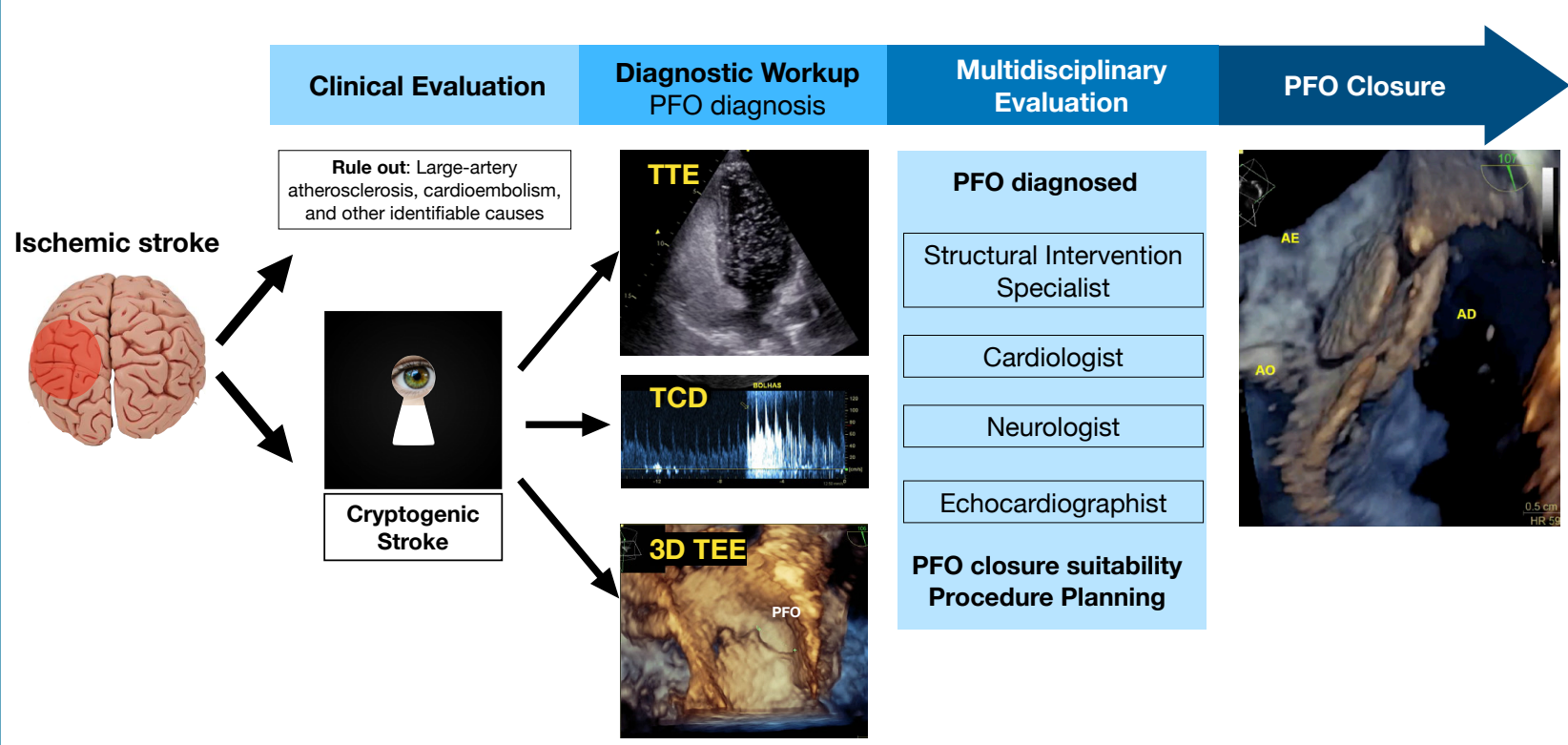

Figure 1 - Flowchart of PFO closure in cryptogenic stroke. PFO = patent foramen ovale, TTE = Transthoracic echocardiogram, TCD $=$ transcranial Doppler, $3 \mathrm{D}$ TEE $=$ three-dimensional transesophageal echocardiogram

and transesophageal study (TEE) using agitated saline solution with adequately performed Valsalva maneuver throughout the steps. TTE will test the quality of contrast arrival and Valsava maneuver will be diagnostic in most cases. TCD will help quantify the shunt by means of a more precise bubble count, and TEE will define the anatomy for interventional planning (Figure 1). Large shunts and high-risk anatomic PFO are well-depicted by three-dimensional echocardiogram, allowing for a better periprocedural guidance and the achievement of optimal results.

In younger patients, who face a longer period of recurrent stroke risk, and in patients with contraindications to long-term anticoagulation, the benefits of transcatheter therapy is less debatable, and contemporary devices have promoted a reduction in the incidence of complications, even though not negligible ${ }^{8}$. Since the publication by Kutty et al., ${ }^{9}$ when available evidence pointed to uncertainty regarding the potential benefits of PFO closure compared to medical treatment alone, some well conducted studies have broken this paradigm, such as the one carried out by Wahl et al., ${ }^{10}$ They enrolled 308 consecutive patients to either undergo PFO closure $(n=150)$ or maintain medical therapy $(n=$ 158), and demonstrated a significant reduction in the composite endpoint of stroke, transient ischemic attack (TIA) and peripheral embolism in the PFO closure group
$(11 \%$ vs $21 \%$, hazard ratio $=0.43 ; 95 \% \mathrm{CI}=0.20-0.94 ; \mathrm{P}=$ 0.033). Since then, new evidence has emerged and data from new studies, using new devices, and well-designed patient selection, have allowed for the establishment of very solid recommendations for this treatment option in selected patients, as stated in the recently updated AAN guidelines ${ }^{3}$. In the present paper, Pereira and cols ${ }^{4}$ also highlight the increased risk of atrial fibrillation in patients undergoing PFO closure (RR for PFO closure, $4.64 ; 95 \% \mathrm{CI}, 2.38$ to $9.01 ; \mathrm{p}<0.01)$, which reinforces the need for adequate selection, as well as careful balance of risk and benefits when indicating this procedure. The choice for intervention should preferably contemplate centers with large expertise and low rates of procedural complications.

In summary, the present study refines the existing evidence for additional risk reduction of PFO closure vs medical treatment alone, through the analysis of less biased data derived from the original clinical trials, as clearly stated by the authors ${ }^{4}$. Considering that many patients are particularly young and may benefit from a long-lasting risk reduction promoted by the intervention, these findings acquire even greater importance. The constant pursue for updated data as science moves forward is invaluable, in light of newer and refined transcatheter techniques and the development of new anticoagulant drugs. 


\section{References}

1. Lechat P, Mas JL, Lascault G, Loron PH, Theard M, Klimczac M, et al. Prevalence of patent foramen ovale in patients with stroke. N Engl J Med. 1988;318(18):1148-52.

2. Saver JL, Mattle HP, Thaler D. Patent Foramen Ovale Closure Versus Medical Therapy for Cryptogenic Ischemic Stroke. A Topical Review. Stroke. 2018; 49(12):1541-8.

3. Pristipino C, Sievert H, D'Ascenzo F, Mas JL, Meier B, et al. European position paper on the management of patients with patent foramen ovale. General approach and left circulation thromboembolism. EuroIntervention. 2019;14(13):1389-402.

4. Messé SR, Gronseth GS, Kent DM, Kizer JR, Homma S. Practice advisory update summary: Patent foramen ovale and secondary stroke prevention. Report of the Guideline Subcommittee of the American Academy of Neurology. Neurology 2020 Apr 29 [Epub ahead of print] DOI: 10.1212/ WNL.0000000000009443

5. Pereira SPP, Nunes A, Santos C, Kasner SE, Nunes JPL. The Role of Patent Foramen Ovale Closure in the Secondary Prevention of Cryptogenic Stroke: a Meta-Analysis Report. Int J Cardiovasc Sci. 2020; 33(4):307-317. DOI: $10.36660 /$ ijcs.20190075
6. Lee PH, Song J-K, Kim JS, Heo R, Lee S, Kim D-H, et al. Cryptogenic Stroke and High-Risk Patent Foramen Ovale. The DEFENSE-PFO Trial. J Am Coll Cardiol. 2018;71(20):2335-42.

7. Mas JL, Derumeaux G, Guillon B, Massardier E, Hosseini H, Mechtouff L, et al. Patent Foramen Ovale Closure or Anticoagulation vs. Antiplatelets after Stroke. N Engl J Med. 2017;377(11):1011-21.

8. Aggeli C. Verveniotis A. Andrikopoulou E. Vavuranakis E. Toutouzas K. Tousoulis D. Echocardiographic features of PFOs and paradoxical embolism: A complicated puzzle. Int J Cardiovasc Imaging. 2018;34(12):1849-61.

9. Kutty S, Sengupta PP, Khandheria BK. Patent foramen ovale: the known and the to be known. J Am Coll Cardiol. 2012;59(19):1665-71.

10. Wahl A, Jüni P, Mono ML, Kalesan B, Praz F. Long-Term Propensity Score-Matched Comparison of Percutaneous Closure of Patent Foramen Ovale With Medical Treatment After Paradoxical Embolism. Circulation. 2012;125(6):803-12 